\title{
Characterization of knitted coils for e-textiles
}

\author{
K. Fobelets, Senior Member, IEEE, K. Thielemans, Senior Member IEEE, A. Mathivanan and C. \\ Papavassiliou, Senior Member IEEE
}

\begin{abstract}
Inductor coils are integrated in many wearable garments for EM wave screening, heating and health monitoring. This paper presents a critical evaluation of the inductor characteristics of circular weft knitted coils for applications in etextiles. Inductors are knitted using circular needles with thin insulated metal wire and yarn knitted together. The resulting helical coils are characterized as a function of number of turns, coil diameter, needle size and insulated metal wire material. The results are compared to wound coils. Simulations of the knitted and wound coils show close agreement with the experimental results and confirm a higher inductance for the knits compared to the wound coils with the same pitch between turns. The parasitic coil capacitance is higher in the knit due to the vertical legs of the stitches, absent in wound coils. Knits with thin $\mathrm{Cu}$ and Litz wires result in flexible and wearable textile coils.
\end{abstract}

Index Terms - Flexible coils, smart textiles, circular weft knit.

\section{INTRODUCTION}

$I^{2}$ NTEGRATION of electrical wiring and electronics within etextiles is the next technological advancement in portable consumer electronics. A large body of work is available covering different approaches to fabricating e-textiles, including printing onto fabrics, embroidery and knitting [1]. The applications range from wearable sensors [2,3], protection [4], heating [5], wireless power transfer [6], electromagnetic shielding [7], healthcare and wellbeing [8], to fashion [9]. Important aspects in e-textiles and wearable electronics are wearability, drape, elasticity and conformity of the fabric. Users' acceptance of novel e-textiles-based clothing is not solely determined by added functionality but also requires comfort and compliance with current fashion. It is therefore important that any electronics integrated within e-fabrics retain fabric flexibility whilst being sufficiently robust to handle everyday wear and regular laundry. These aspects are amongst the main challenges in enabling a breakthrough of etextiles and wearable electronics in the consumer market and impose restrictions on the operational quality of the electronics integrated in the fabric.

Embroidering, weaving and knitting are traditional techniques applied in the textile industry. Embroidery is a technique applied at the end of the fabrication of the fabric, and is often used as decoration. The other two techniques

Manuscript received March 2019.

A. Mathavanan acknowledges financial support from the UROP placement scheme of Imperial College London.

K. Fobelets, C. Papavassiliou and A. Mathavanan are with the Electrical and Electronic Engineering Department, Imperial College London,

Exhibition Road, SW7 2AZ London, UK. (e-mail corresponding author: k.fobelets@imperial.ac.uk)

K. Thielemans is with the Institute of Nuclear Medicine, UCL, UCL Hospital, NW1 2BU London, UK. include conductive yarn within the fabric making process. The advantage of knitting over weaving is the elasticity knitting gives to the fabric due to the horseshoe shaped interlinked stiches $[10,11]$.

Coils have been integrated into knits in different ways. One example is where stainless steel yarn is used for knitting, resulting in a cylindrical conductive sheet [8]. The implementation gives a relatively small inductance and large resistance value that both change with changing diameter. Additional fabric layers need to be added to insulate the conductive knit from the body when worn. Another example is where an insulated conductive wire is inlaid into a knit without being part of the knitted stitches [12]. This approach allows coils with multiple windings and thus higher inductance values but does not allow changes in the diameter of the coil. In this manuscript, the insulated conductive wire is knitted with the yarn and thus forms part of the stitches. This gives a coil with an inductance that can be tuned by the number of turns (knitted rows) but also gives a flexible cylinder that allows changes in diameter of the coil. Implementations of this type of knits are particularly interesting for respiration and posture monitoring [8, 13] where the flexibility of the knit is the main parameter in the application.

Two types of knits exist, weft and warp knits. Weft knitting requires only a single yarn and when applied to circular knitting, produces a tubular structure with yarn that runs continuously from start to finish along the circumference of the cylinder. It results in a fabric with very high flexibility and stretch. Warp knitting requires each end of a row in the knit to be fed with separate yarn feeds over the whole width of the fabric. Thus it does not generate a continuously running yarn along the circumference of the cylinder, but it allows more customization and is less prone to runs than weft knits. Only circular weft knitting is suitable for the fabrication of helical coils with multiple turns. This is illustrated in fig. 1. In a circular knit, the stitch ending indicated with a hexagon or circle continues into the stitch identified with the same shape. As a consequence, the current flowing through conductive yarn in the "horizontal" direction is similar to that flowing through a classical helical coil and the magnetic fields due to the currents flowing in the "horizontal" directions, sum. The parts of the stitch in the "vertical" direction create opposite currents with magnetic fields that approximately cancel. These tubular shapes can be easily integrated in clothing, e.g. sleeves, trousers and sweaters. Planar circular weft-knitted coils are also possible but are less convenient for integration into knitted clothing that is based on more rectangular shapes. For planar coils integrated into clothing, printing or embroidering might be better alternatives. 


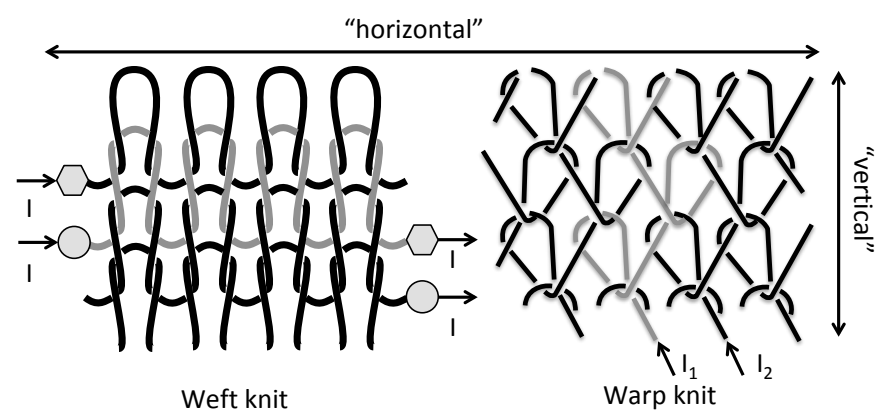

Fig. 1. Left: weft knit in which the stitches are running horizontally. The circular and hexagon shapes indicate where the stitches come together in a helical shape. The currents with arrows indicate continuity of the current through the knit. Right: warp knit. The stitches run zigzag and the different zigzags are connected to different yarn feeds, indicated by two currents $I_{1}$ and $\mathrm{I}_{2}$. "Horizontal" are those parts of the loop in the horizontal direction along the circumference of the knit. "Vertical" are the perpendicular parts of the loop along the length of the knit.

In this manuscript, we present the measured and simulated self-inductance, equivalent resistance and capacitance of weft, hand-knitted, helical coils. The coils are made by simultaneously knitting cotton yarn and insulated conductive wire. The conductive wires used are: soft $\mathrm{Cu}$ for jewelry fabrication with a diameter of $250 \mu \mathrm{m}$ [14], $\mathrm{Cu}$ for coil windings with a diameter $\sim 110 \mu \mathrm{m}$ (gauge $=0.048)$ [15], and $80 / 20 \mathrm{Cu} / \mathrm{Ag}$ Litz wire with a total diameter of $\sim 150 \mu \mathrm{m} \mathrm{[16].}$ From an aesthetic and wearability point of view, the Litz wire integrates best, not hindering drape nor flexibility. The 250 $\mu \mathrm{m} \mathrm{Cu}$ wire has the lowest resistivity but results in unwearable fabrics. Current development of conductive yarn, based on hybrid yarns and composites, will open up a wider range of conductive material that can be used in the future $[17,18]$.

The manuscript is organized as follows. In Section II, the simulation approach for the knitted coil is described. In Section III, the self-inductance of the knitted helical coil is measured as a function of number of turns and compared to wound coils and simulations. In Section IV, the influence of the diameter, the needle size and the conductive wire type is presented. Section V summarizes the results.

\section{SimUlations OF THE INDUCTANCE OF THE KNIT}

Simulations to estimate the self-inductance of the wound and knitted coils are based on the theory presented in [19], and take the horseshoe geometry of the stitches into account. In [19] the self-inductance of a coil with smooth loops and a length much larger than the diameter of the wire, was extracted from the Neumann expression for mutual inductance. The equation for the self-inductance is based on a double loop integral along the curve of the wire and was found to give excellent approximate results for complex curves. The equation of the self-inductance is [19]:

$$
L \approx \frac{\mu_{0}}{4 \pi}\left(\oiint \frac{d x_{1} \cdot d x_{2}}{\left|x_{1}-x_{2}\right|}\right)_{\left|s_{1}-s_{2}\right|>a / 2}+\frac{\mu_{0}}{4 \pi} l Y+\cdots
$$

with $a$ the wire diameter, $l$ its length, $s$ measures the length along the wire axis and $Y$ is a parameter that takes the current distribution within the wire into account. Test simulations of knitted coils show that the magnitude of the second term is approximately 100 times smaller than the first term and is thus negligible.
The model of a stitch is based on the parameterization of a knitted stitch with a geometry proposed in [20] (see dots in fig. 2 inset 2). Seven points define a stitch with one point at each side shared with the neighboring stitches. Mathematica [21] is used to generate the rounded horseshoe geometry of a stitch by connecting these points using cubic spline interpolation. The single stitch is repeated to obtain the number of stitches needed along one winding. Wrapping the resulting function of the stitches along a helix gives a knitted helical coil (see fig. 2).

Numerical integration of eq. (1) for different stitch parameters is also done in Mathematica as it computes the derivative of the spline function analytically. Implementation of the double integral in eq. (1) needs some care. Simply setting the integrant to zero when $\left|\boldsymbol{x}_{1}-\boldsymbol{x}_{2}\right|<a / 2$ leads to very slow convergence as Mathematica's integration routine detects a discontinuity. Instead, the integral was computed using numerically determined integration boundaries. The use of discrete points to define the stiches requires two additional points in front of the $1^{\text {st }}$ stitch and after the last stitch to allow for correct differentiation of the numerator in eq. (1). These points then need to be removed in the final curve calculation. The stitch was modelled with the top and bottom points on a smaller radius than the middle points to reflect a realistic knit where the wire of the stitches in the consecutive row goes in front of those of the previous row (see fig. 1 and fig. 2 inset 2). The movement will be larger for thicker yarn (larger needle size). Note that $\left|\boldsymbol{x}_{1}-\boldsymbol{x}_{2}\right|>a / 2$ if the points are on different rows. To simulate the real knits, the measured height of the stitches is implemented together with the number of stitches knitted per row.

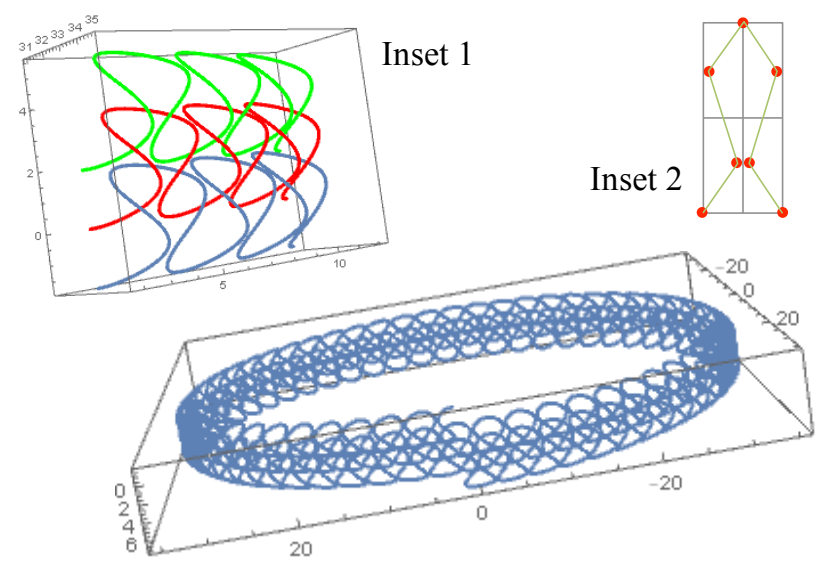

Fig. 2. Simulated (Mathematica) geometry of 3 knitted rows. The dimensions are given in $\mathrm{mm}$. Top-left shows a zoomed version on 3 stitches in each row (each row displayed with a different color for clarity). Inset top-right: parameterization points of one stitch. The dots are the points that define the horseshoe shape of the stitch. The line connects these dots via a cubic spline interpolation.

The wound coil without stitches but with turns at a distance of the stitch height (pitch) is also simulated using eq. (1).

The results of the simulations are presented on the same figures as those for the measurements that are given in the following section. 


\section{SELF-INDUCTANCE OF KNITTED COILS}

Helical coils with a diameter $D=6.7 \mathrm{~cm}$ - suitable for cuffs in a garment - are weft knitted using circular needles and $\mathrm{Cu}$ wire of gauge 0.048 . The needles are $2 \mathrm{~mm}$ in diameter giving a measured stitch height of $\sim 1.8 \mathrm{~mm}$ with 60 stitches for the given circumference. Ten different cuffs are knitted with the number of turns increasing from $N=1$ to 10 (fig. 3a). A cardboard cylinder supports the cuffs during measurements. Simple wound coils $(N=1-9)$ are made by closely winding the $\mathrm{Cu}$ wire on the cardboard cylinder with the same diameter as the inner diameter of the knitted cuffs and with the pitch between turns equal to the stitch height, $1.8 \mathrm{~mm}$. Finally, a coil $(N=1-10)$ is closely wound on the cardboard cylinder. The ends of the $\mathrm{Cu}$ wire are soldered to appropriate contacts to connect to the equipment.

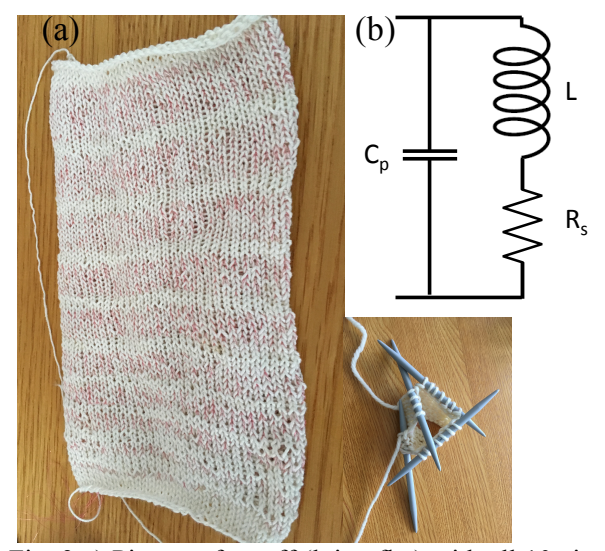

Fig. 3 a) Picture of a cuff (lying flat) with all 10 circular knitted coils $N=1-$ 10 , using $2 \mathrm{~mm}$ needles and 0.048 gauge insulated $\mathrm{Cu}$ wire. Inset: shows the start of the circular knit with 4 needles. Needles are $10 \mathrm{~mm}$ diameter in this picture. b) Equivalent circuit of a helical coil. $L$ : self-inductance, $R_{s}$ : series resistance, $C_{p}$ : parallel capacitance.

S11 measurements are carried out using a HP8753D Network Analyzer $(10 \mathrm{kHz}-6 \mathrm{GHz})$ in a frequency range from $100 \mathrm{kHz}$ to $50 \mathrm{MHz}$. The analyzer is calibrated with open, short and $50 \Omega$ connections. Results are also checked using a Wayne Kerr Precision Impedance Analyzer 6500B (20 $\mathrm{Hz}-10 \mathrm{MHz}$ ). The analyzer calibration is verified with a 100 $\mathrm{pF}$ capacitor and $100 \Omega$ resistor.

The values of self-inductance $L$, equivalent resistance $R_{s}$ and capacitance $C_{p}$ are extracted from the measurements by assuming that the equivalent circuit given in fig. $3 \mathrm{~b}$ is valid. This assumes that the knit behaves in a similar way to a wound coil. The results for $L$ and $R_{s}$ of both measurement approaches are in agreement. The result of the extraction of the self-inductance from the S11 parameters is given in fig. 4, together with the simulation results. Fig. 4 shows that the knitted coil's behavior lies between that of the closely wound coil and the coil wound with the same pitch height as the knit, which generates the same overall coil length as the knit. This shows that the knit does indeed create an inductor with an inductance value mainly determined by the number of turns and the diameter of the coil.

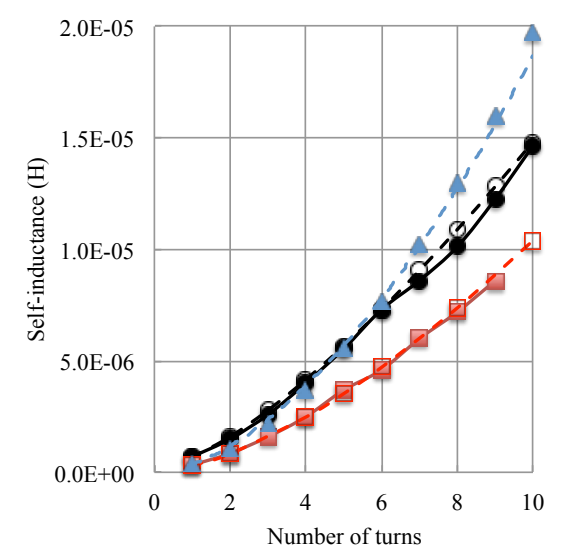

Fig. 4. Closely wound coil (triangles), dashed line is a power fit to the measurements (power factor is 1.72). Squares: wound coil with $1.8 \mathrm{~mm}$ pitch. Circles: knitted coil. Full markers and lines are the measurements, dashed lines and open markers are the simulations (eq. 1). Knitting needles are $2 \mathrm{~mm}$, diameter of the coil is $6.7 \mathrm{~cm}$ and 0.048 gauge insulated $\mathrm{Cu}$ wire was used.

The resistance, $R$ of the coil (fig. 5a) is directly proportional to the total length of the wire and changes linearly with number of turns $N: R=a N+b$ with $a=0.3,0.3$ and 1 and $b=$ $0.3,0.09$ and 0.1 for the closely wound, wound at pitch height and knitted coil, respectively. The constant, $b$ in the linear fit gives the average contact resistance due to the wire length needed to connect to the equipment and the resistance of soldering to the insulated wire. The main drawback of a knitted coil is the higher overall resistance since knitting uses more wire than windings. The total length of the knitted yarn for $N=10$ is approximately $\sim 9 \mathrm{~m}$, whilst simple turns only take $\sim 2.2 \mathrm{~m}$ of wire length. Indeed, maintaining the flexibility of the garment comes at a cost of increased series resistance and a reduction of the inductor quality factor, $Q$. In fig. $5 \mathrm{~b}$ the quality factor of the 3 different types of coils is plotted at a frequency of $f=6 \mathrm{MHz}$, calculated following the equation: $Q=\frac{2 \pi f L}{R_{S}}$
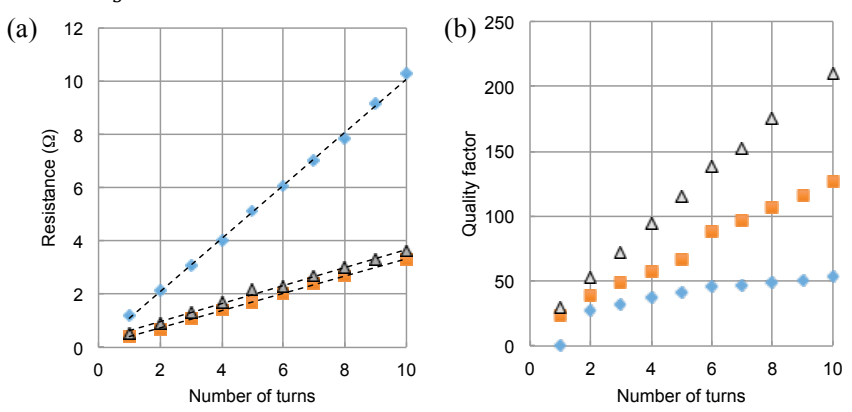

Fig. 5: a) Resistance as a function of number of turns. Markers are as in fig. 4. The dashed lines are linear fits to the measurements. b) The quality factor, $Q$ at $6 \mathrm{MHz}$ as a function of number of turns, $N$ for wound coil (triangles), inlaid coils into knit (squares) and knitted coils (diamonds).

In [22], the quality factor, $Q$ is reported at $200 \mathrm{kHz}$. For the knitted coil with $N=10, \mathrm{Q} \approx 1.6$ at $200 \mathrm{kHz}$. This value is comparable to the results of the screen-printed coils in [22] but lower than those considered useful for wireless power transfer.

The parasitic capacitance, $C_{p}$, can be derived from the selfresonance of the parallel LCR circuit:

$\omega_{\text {res }}=\frac{1}{L} \sqrt{\frac{L}{C_{p}}-R_{S}^{2}} \approx \frac{1}{\sqrt{L C_{p}}}$ for $R_{s}$ small. 
Fig. 6a gives $C_{p}$ based on eq. (6) for both the knitted and wound coil for different values of $N$.

The parasitic capacitance tends to be higher for the knitted compared to the wound coil due to the different distributed capacitance network for the two approaches. For the knit the network consists of a combination of parallel and series connections. For the wound coils only parallel capacitors occur. The value of $C_{p}$ is approximately constant as a function of number of turns and for constant coil circumference.
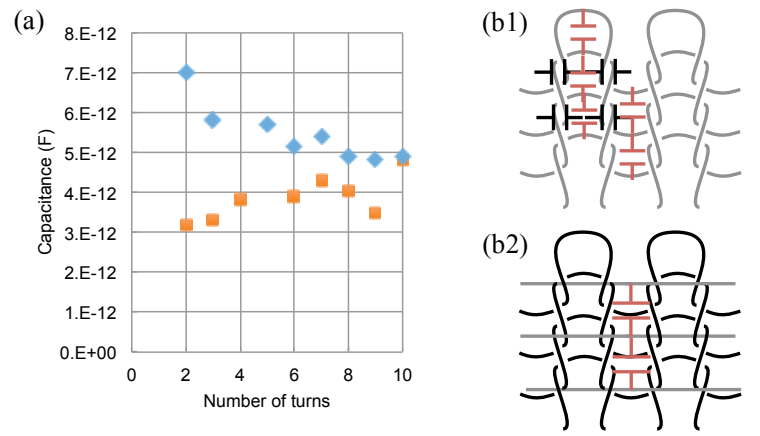

Fig. 6. a) The parallel capacitance extracted from the frequency at which the impedance of the coil is maximal, as a function of number of turns, for the knitted (diamonds) and wound at pitch height (squares) coils. b1) distributed capacitors in the knitted system. b2) Distributed capacitances in the wound system. Grey: presence of metal wire.

\section{INFLUENCE OF OTHER PARAMETERS}

This section presents the study of the influence of the distance between rows in which the conductive wire is included, the diameter of the coil, the type of conductive wire used and the needle size. All measurements are carried out with $N=5, D=6.7 \mathrm{~cm}$ and needle size of $2 \mathrm{~mm}$ unless otherwise indicated.
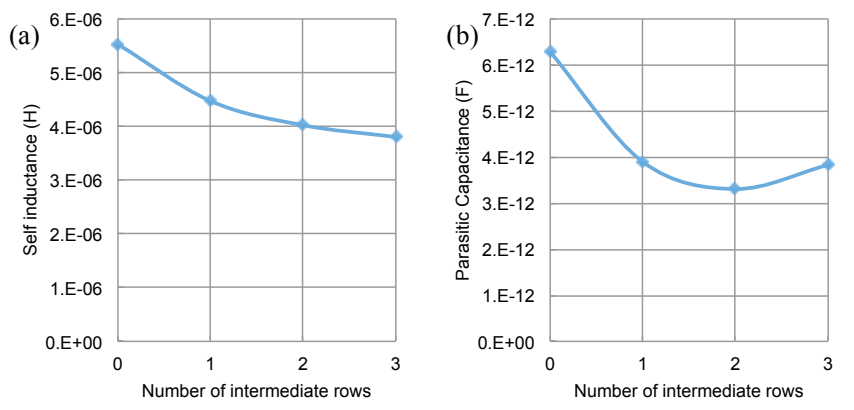

Fig. 7. a) Self-inductance, b) capacitance as a function of number of rows in which metal wires are excluded from the stitches. The cuff diameter is $6.7 \mathrm{~cm}$ and the total number of windings with metal included is $N=5$.

The influence of the distance of the conductive wires in the knit is investigated by skipping the metal wire for 1,2 , or 3 rows. This positions the horizontal part of the knitted wire at ever increasing distances whilst still maintaining wire continuity. Fig. 7 confirms that the self-inductance lowers with a wider separation between the wires due to reduced magnetic coupling. The reduction in parallel capacitance seems to suggest an inversely proportional relation to the separation of the horizontally lying parts of the stitched wires, and thus also suggest that this separation controls $C_{p}$.
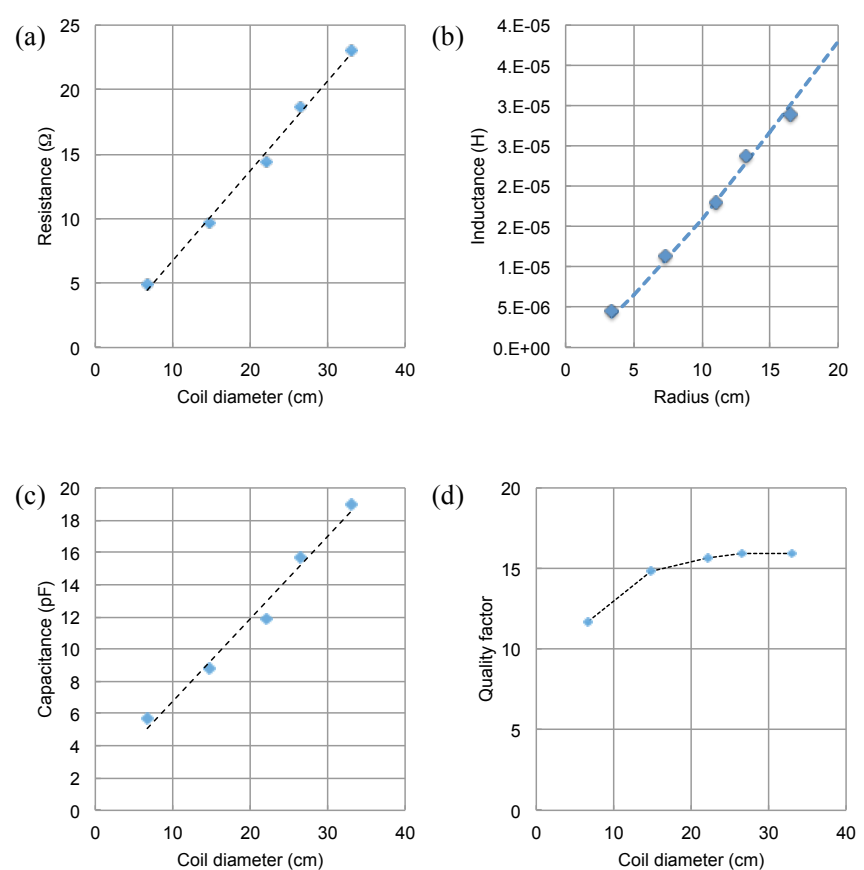

Fig. 8 a) Resistance, b) self-inductance, c) capacitance and d) quality factor at $6 \mathrm{MHz}$ as a function of coil diameter for $N=5$, needle size $2 \mathrm{~mm}$. Dashed lines are guides to the eye.

Fig. 8 shows the parameters of the knitted coil as a function of coil diameter. The increased diameter is achieved by increasing the number of stitches $(60,120,180,240$ and 300 stitches for increasing diameters). Some of the diameters chosen are those suitable for a cuff, trouser leg and waist of a garment. The self-inductance increases linearly with diameter. A linear variation is attractive for applications where changes in cross sectional area are measured $[8,13]$. The parasitic capacitance also increases linearly with diameter suggesting that $C_{p}$ is approximately proportional to the circumference of the coil. The increased resistance of the coil with increasing diameter causes the quality factor to saturate for higher diameters.

In order to improve the quality factor of the coils, other conductive wires can be used. In addition to the 0.048-gauge $\mathrm{Cu}$ wire, we used $250 \mu \mathrm{m}$ diameter soft insulated $\mathrm{Cu}$ jewelry wire and insulated $80 / 20 \mathrm{Cu} / \mathrm{Ag} \mathrm{Litz}$ wire with a diameter of $\sim 150 \mu \mathrm{m}$. The results in fig. 9 show that the self-inductance is approximately independent of wire material, although the thinnest wire seems to generate a slightly higher inductance, as expected from eq. (1). The difference in resistance however is large with, as expected, the resistance of the thickest $\mathrm{Cu}$ wire lowest, leading to a higher quality factor. The knits with the thicker $\mathrm{Cu}$ wire however resulted in unwearable material. The Litz wire generated the best wearable material with good stretch and drape.

The results in fig. 9 also include the impact of the needle size (the yarn gauge used is consistent with the needle size). The experiments suggest that the influence of the needle size on the self-inductance and resistance is negligible for the given diameter and turns $(D=6.7 \mathrm{~cm}$ and $N=5)$. The Mathematica simulations in fig. 9 d) support these experimental conclusions but suggest a somewhat weaker dependence of the selfinductance on number of turns for large needle size. 

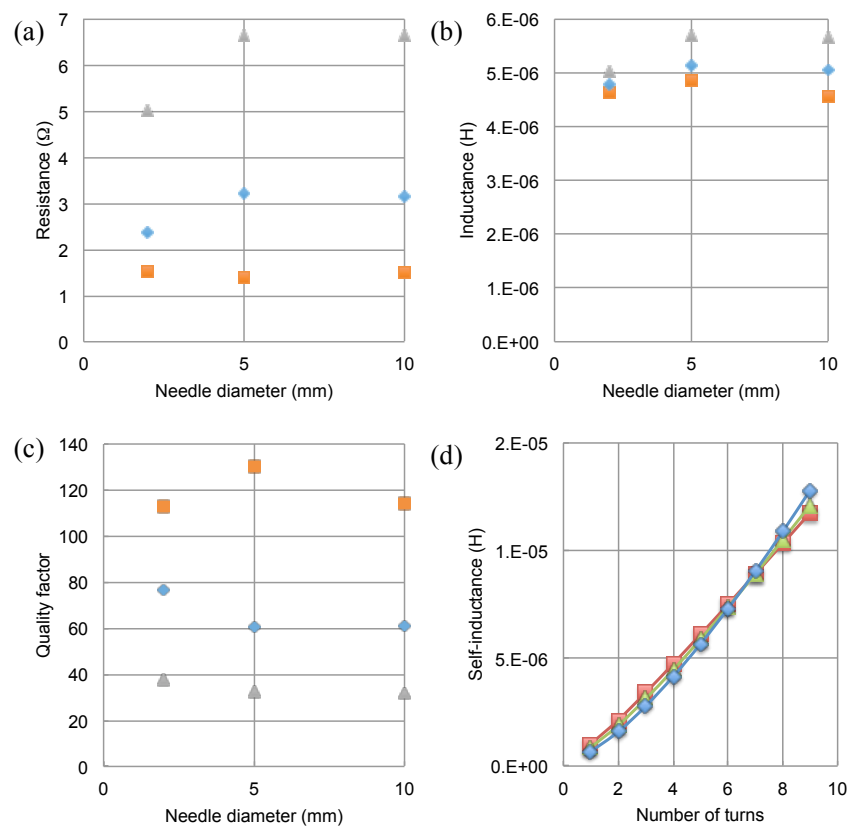

Fig. 9. Influence of needle size and conductive wire for $N=5$ and $D=6.7 \mathrm{~cm}$. a) Series resistance, b) self-inductance and c) quality factor at $6 \mathrm{MHz}$ as a function of wire type and knitting needle diameter. Squares: $250 \mu \mathrm{m} \mathrm{Cu}$, diamonds: Litz wire, triangle: $110 \mu \mathrm{m} \mathrm{Cu}$. (d) Simulations of the selfinductance as a function of needle diameter. Needle size: diamonds $2 \mathrm{~mm}$; triangles $5 \mathrm{~mm}$ and squares $10 \mathrm{~mm}$.

\section{CONCLUSION}

In this work the feasibility for incorporating coils in knitted fabrics by knitting a thin insulated conducting wire together with normal yarn was investigated. We confirmed that weft round-knitted tubular structures resulted in coils with a selfinductance similar to wound coils. The variation of the selfinductance as a function of number of turns and needle size was simulated in Mathematica and showed a close match between simulations and measurements, providing confidence in the expeimental set-up and showing that the simulations can be used for optimisation of a design in future work.

The parasitic capacitance, $C_{p}$ of the weft knitted coil is higher than that found for a wound coil with the same pitch as the knit and for the same number of turns. Whilst the parasitic capacitance remains nearly constant as a function of number of turns, it increases as a function of coil diameter, suggesting that the parts of the stitches along the diameter of the coil have the largest influence on $C_{p}$.

The resistance of the knitted coils is higher than that of wound coils as the knit requires more wire length. This results in a trade-off between wearability and coil quality factor.

The diameter of the knitting needles does not have an appreciable impact on the self-inductance. This suggests that the main parameters that determine the self-inductance of the knitted coils are the diameter of the coil and the number of turns. The simulations support these conclusions.

In the proposed knitted coil, the flexibility of the garment is greater than that of an inlaid coil allowing the garment to stretch. In addition, the inductance changes linearly with diameter in contrast to the approach where the knits are based on electrically connected stitches. Both features are advantageous for sensing diameter changes as used in e.g. plethysmography [23].

Similar to other approaches in e-textiles, the requirement for wearability imposes limitations on the performance of the integrated electric components. In this case the resistance is higher than wound coils and thus leads to a lower quality factor. Reducing the resistance will be essential for applications in wireless power transfer. Litz wire gives the best results in applications where both wearability and quality factor are important. Further improvements can be implemented with Litz wires with higher conductivity using a higher overall diameter or increasing the $\mathrm{Ag}$ contents in the wires.

\section{REFERENCES}

[1] T. Hughes-Riley, T. Dias and C. Cork, "A Historical Review of the Development of Electronic Textiles", Fibers 6, 34 (15 pp), 2018; DOI:10.3390/fib6020034

[2] S. D. Tohidi, A. Zille, A. P. Catarino, and A. M. Rocha, "Effects of Base Fabric Parameters on the Electro-Mechanical Behavior of Piezoresistive Knitted Sensors", IEEE Sensors Journal 18(11), pp. 4529-4535, 2018. DOI: 10.1109/JSEN.2018.2826056

[3] M. Stoppa and A. Chiolerio, "Wearable Electronics and Smart Textiles: A Critical Review", Sensors 14, pp. 11957-11992, 2014; DOI: $10.3390 / \mathrm{s} 140711957$

C. Gonçalves, A. Ferreira da Silva, J. Gomes, and R. Simoes, "Wearable E-Textile Technologies: A Review on Sensors, Actuators and Control Elements", Inventions, 3(1), 14 (pp. 13), 2018; DOI:10.3390/inventions3010014

[4] A. Gugliuzza, and E. Drioli, "A review on membrane engineering for innovation in wearable fabrics and protective textiles", Journal of Membrane Science 446, pp. 350-375, 2013.

[5] L. Zhang, M. Baima, and T.L. Andrew, "Transforming Commercial Textiles and Threads into Sewable and Weavable Electric Heaters", ACS Appl. Mater. Interfaces 9, pp. 32299-32307, 2017. DOI: 10.1021/acsami.7b10514

[6] D. Zhu, N. J. Grabham, L.R. Clare, B.H. Stark, and S.P.. Beeby, "Inductive Power Transfer in E-Textile Applications: Reducing the Effects of Coil Misalignment", IEEE Wireless Power Transfer Conference (WPTC) 13-15 May 2015. DOI: 10.1109/WPT.2015.7140116

[7] Z-C Yu, J-F Zhang, C-W Lou, H-L He, A-P Chen and J-H Lin, "Determination of electromagnetic shielding and antibacterial properties of multifunctional warp-knitted fabrics", The Journal of The Textile Institute 106(11), pp. 1203-1211, 2015.

[8] M. Catrysse, R. Puers, C. Hertleer, L. Van Langenhove, H. van Egmond, D. Matthys, "Towards the integration of textile sensors in a wireless monitoring suit", Sensors and Actuators A 114, pp. 302-311, 2004. DOI: doi.org/10.1016/j.sna.2003.10.071

[9] https://www.mrs.org/spring-2018-wearables-in-smart-fabrics-fashionshow

[10] W. Zeng, L. Shu, Q. Li, S. Chen, F. Wang, and X-M Tao, "Fiber-Based Wearable Electronics: A Review of Materials, Fabrication, Devices, and Applications", Adv. Mater. 26, pp. 5310-5336, 2014. DOI: 10.1002/adma.201400633.

[11] L.M. Castano and A.B. Flatau, "Smart fabric sensors and e-textile technologies: a review", Smart Mater. Struct. 23, 053001 (27pp), 2014. DOI:10.1088/0964-1726/23/5/053001

[12] Y. Zhu, "A Wireless Power Transfer Wearable Garment", MSc thesis North Carolina State University (2017) https://repository.lib.ncsu.edu/bitstream/handle/1840.20/33548/etd.pdf? sequence $=1$

[13] E. Sardini, M. Serpelloni, and V. Pasqui, "Wireless Wearable T-Shirt for Posture Monitoring During Rehabilitation Exercises", IEEE Trans Instrumentation and Measurements 64(2), pp. 439-448, 2015

[14] www.mahliqa.co.uk

[15] Lewcos, UK

[16] Kindly donated by Textile-Wire, Switzerland 
[17] Y. Huang, H. Hu, Y. Huang, M. Zhu, W. Men, C. Liu, Z. Pei, C. Hao, Z. Wang, and C. Zhi, "From Industrially Weavable and Knittable Highly Conductive Yarns to Large Wearable Energy Storage Textiles", ACSNano 9(5), pp. 4766-4775, 2015.

[18] S. Maity and A. Chatterjee, "Conductive polymer-based electroconductive textile composites for electromagnetic interference shielding: A review", Journal of Industrial Textiles 47(8), pp. 22282252, 2018.

[19] R. Dengler, "Self inductance of a wire loop as a curve integral", $A d v$. Electromagnetics 5(1), pp. 1-8, 2016.

[20] Z. Lu, and G. Jiang, "Rapid simulation of flat knitting loops based on the yarn texture and loop geometrical model", AUTEX Research Journal 17(2), pp. 103-110, 2017.

[21] Mathematica, Wolfram.com.

[22] N.J. Grabham, Y. Li, L.R. Clare, B.H. Stark, and S.P. Beeby, "Fabrication Techniques for Manufacturing Flexible Coils on Textiles for Inductive Power Transfer", IEEE Sensors Journal 18(6), 2599 2606, 2018.

[23] M. Folke, L. Cernerud, M. Ekström, B. Hök, "Critical review of noninvasive respiratory monitoring in medical care", Medical and Biological Engineering and Computing 41(4), pp 377-383 2003. 